\title{
Scintillation properties of solution-grown trans-stilbene single crystals
}

\author{
Natalia Zaitseva ${ }^{\mathrm{a}, *}$, Andrew Glenn ${ }^{\mathrm{a}}$, Leslie Carman ${ }^{\mathrm{a}}$, H. Paul Martinez ${ }^{\mathrm{a}}$, Robert Hatarik ${ }^{\mathrm{a}}$, \\ Helmut Klapper ${ }^{\mathrm{b}}$, and Stephen Payne ${ }^{\mathrm{a}}$ \\ ${ }^{a}$ Lawrence Livermore National Laboratory, 7000 East Avenue, Livermore, CA 94551, USA \\ ${ }^{b}$ Institut für Kristallographie, Jägerstraße 17-19, D-52066 Aachen, Germany,
}

\begin{abstract}
The scintillation properties of trans-stilbene crystals grown for the first time by application of the solution growth technique to the scale of $10 \mathrm{~cm}$ are reported. Measurements of the scintillation light output, pulse shape discrimination, and neutron detection efficiency were made with sets of crystals cut as $50 \mathrm{~cm}$ diameter cylinders of different lengths from 0.3 to $10 \mathrm{~cm}$. Comparison to liquid scintillators and traditional melt-grown stilbene showed that at increasing sizes new solution-grown crystals exhibit better scintillation performance that makes them promising for use in large scale neutron detectors. Results are discussed in relation to structural imperfections attributed to different methods of growth.
\end{abstract}

Keywords. Trans-stilbene; Solution growth; Organic crystals; Organic scintillator; Pulse shape discrimination; Neutron detection.

Corresponding author.

E-mail address: zaitseva1@1lnl.gov (N. Zaitseva) 


\section{Introduction}

Trans-stibene (diphenylethylene) is a molecular organic crystal built of aromatic hydrocarbon molecules, $\mathrm{C}_{14} \mathrm{H}_{12}$. Trans-stilbene single crystals were used in initial studies $[1,2]$ that led to development of the pulse shape discrimination (PSD) technique [3] applied for separation of scintillation signals produced by different types of incident radiation. According to the literature data, it has scintillation efficiency lower than that of anthracene crystals [4], but its remarkable ability to discriminate between particles of different types [5] made it known as "one of the most useful organic scintillators" [6]. Good PSD properties combined with a high content of hydrogen in its composition make stilbene particularly useful for direct detection of fast and high-energy neutrons discriminated from strong gamma radiation background. Multiple studies $[3,7-10]$ have proven that stilbene crystals are better suited for fast neutron spectroscopy and neutron-gamma discrimination than any other organic scintillators. However, when it comes to the design of detectors, which require large-volumes of scintillators [11, 12], organic liquid scintillators are considered the preferred materials due to their low cost and easy availability. In spite of more than a six-decade history of development, stilbene single crystals are rarely produced at dimensions exceeding $10 \mathrm{~cm}$, having more common sizes not larger than 6-8 cm [13]. The relatively high cost and limited availability of stilbene crystals stimulated the recent development of new types of solid-state PSD materials, such as composite [13] or plastic [14] scintillators that can be produced in larger volumes and at lower cost. However, based on the current data, it is hard to expect that either composites or plastics can be manufactured in the near future with PSD or spectroscopic properties equal to those of the stilbene crystal. To increase the availability and volume of detector materials while decreasing associated costs, more efficient methods of crystal growth can be considered. Traditionally, trans-stilbene has 
been grown by melt (Bridgman) techniques, which are more suitable for isometric crystals such as NaI, rather than low-symmetry organic crystals with the monoclinic structure of stilbene (space group $\mathrm{P} 2{ }_{1} / \mathrm{a}[15]$ ). Since stilbene is soluble in many organic solvents, a possible option is the application of solution growth methods. A specific feature of solution growth, which produces naturally faceted crystals, is the absence of temperature gradients and mechanical restrictions imposed on the growing crystal by crucible walls. Low temperature and simplicity of equipment make solution growth less expensive compared to most melt-growth techniques. An additional advantage is introduced by easier scaling that can be achieved by solution growth, independent of the structural symmetry of growing crystals [16]. Our recent publication [17] described the successful growth of 10-cm scale stilbene crystals of high optical quality from organic solvents. In this paper, we report the scintillation properties of these crystals. Measurements have been made with samples of different dimensions to obtain data that can be of interest for instrument applications, while producing the first consistent studies of trans-stilbene properties at increasing size up to $10 \mathrm{~cm}$. To help put these measurements in perspective for potential end users, the results were referenced to similar measurements made with commercially purchased stilbene crystals produced by traditional melt-growth technique and, in some cases, to the commonly used liquid scintillators of the same size.

\section{Experimental methods}

Crystals were grown by the temperature reduction method from stilbene solutions in anisole. Details of the crystal growth were described in a previous publication [17]. A part of the 
publication was focused on the challenges of material purification. In particular, it showed that none of stilbene raw materials available from commercial vendors around the world was appropriate for production of stilbene single crystals with high scintillation efficiency. The light output of first solution-grown crystals obtained using such materials was 2-3 times lower than that of melt-grown crystals produced by the Ukrainian Institute of Scintillation Materials and distributed in the US by ScintiTech [18]. The problem was resolved in collaboration with ProChem Inc. [19] which recently started commercial production of high-purity stilbene material used for crystal growth in the present studies.

Solution-grown samples were cut from the initial faceted crystals grown to dimensions sufficient to obtain cylinders with $5.1 \mathrm{~cm}$ in diameter and different lengths from 3 to $\sim 100 \mathrm{~mm}$. Non-encapsulated melt-grown cylinders of the same diameter were available from the commercial vendor only at sizes not exceeding $32 \mathrm{~mm}$ in length. Crystals of larger dimensions (50 and $75 \mathrm{~mm}$ ) were initially purchased as full detectors packaged in Al containers. For more accurate comparison, the containers were carefully cut to remove bare crystal cylinders, which then were wrapped in the same way as their solution-grown counterparts. Unfortunately, the crystals removed from the containers remained intact for only a few days, and the light output measurements were performed during this time. Eventual development of severe cracks made them unsuitable for further characterization. As a result, all measurements, with the exception of the scintillation light output, were made with the following sets of melt-grown crystals: bare crystals with a length of $7.5 \mathrm{~mm}, 24 \mathrm{~mm}, 32 \mathrm{~mm}$, and encapsulated (49 and $75 \mathrm{~mm}$ long).

All cylinders used in the measurements were oriented along the crystallographic bdirection. Cylinders wrapped with Teflon film were coupled through a thin layer of optical grease to a 2-inch $(5.1 \mathrm{~cm})$ Hamamatsu R6231-100-SEL photomultiplier tube (PMT). The 
scintillation light output (LO) was evaluated from the position of the Compton edge in the ${ }^{137} \mathrm{Cs}$ spectra, in which $500 \mathrm{keV}_{\mathrm{ee}}$ was defined by $50 \%$ of the Compton edge peak. It should be noted here that measurement of response functions for different type of radiation was out of scope of this work, so all energy values below are expressed in electron-equivalent units. The approximate estimation of corresponding recoil proton energies was made using quenching factors reported for stilbene crystals in previous studies [20,21].

Neutron detection properties were studied, as described previously $[14,22]$ using a ${ }^{252} \mathrm{Cf}$ source. The signals collected at the PMT anode were recorded using a 14-bit high-resolution waveform CompuScope 14200 digitizer with a sampling rate of 200MS/s. The ability of scintillator samples to discriminate between neutrons and gamma rays was evaluated using the charge integration method. The baseline, calculated using 100 pre-trigger samples (500 ns), was subtracted from the waveforms. The waveforms were numerically integrated over two time intervals: $\Delta t_{\text {Total }}$ and $\Delta t_{\text {Tail }}$, corresponding to the total charge $\left(Q_{\text {Total }}\right)$ and the delayed component $\left(\mathrm{Q}_{\text {Tail }}\right)$ of the signal, respectively. The value of the ratio of charge, $R=Q_{\text {Tail }} / Q_{\text {Total }}$, for the two time intervals indicated whether the considered event was likely produced by a neutron (high $\mathrm{R}$ value) or a gamma ray (small $R$ value). Quantitative evaluation of PSD was made using Figures of Merit $F o M=S /\left(\delta_{\text {gamma }}+\delta_{\text {neutron }}\right)$, where $S$ is the separation between gamma and neutron peaks, and $\delta_{\text {gamma }}$ and $\delta_{\text {neutron }}$ are full width at half maximum (FWHM) of the corresponding peaks. The mean and FWHM values for the gamma and neutron distributions were extracted from Gaussian fits. In general, good PSD performance was achieved with tail integration, $Q_{\text {Tail }}$, beginning 40 to $50 \mathrm{~ns}$ after the pulse peak and ending after 400 to $600 \mathrm{~ns}$. $Q_{\text {Total }}$ started $75 \mathrm{~ns}$ before the peak and ended at the same time as $Q_{\text {Tail }}$. More specific integration times are given with the reported 
results below. The integration windows for individual energy ranges were selected based on the highest value of FoM calculated by variations of start and end integration times.

Measurements of the neutron detection efficiency were made by exposing scintillator samples to an unshielded NIST traceable ${ }^{252} \mathrm{Cf}$ source, model CF230140150U produced by Eckert \& Ziegler, with a stated neutron emission rate of 604,000 neutrons/s as of October 1st, 2011. The activity of the source was calculated for the time of the measurements using a 2.645 year half-life. The source was placed $75 \mathrm{~cm}$ from the face of the scintillators to produce a flux approximately perpendicular to the face of the detector. The measured mass and sample length were combined with material density to calculate a cross section correction for each sample to normalize the efficiency to a $20 \mathrm{~cm}^{2}$ cross section $(2.83 \mathrm{e}-2 \%$ geometric acceptance). These corrections were on the order of a few percent. Timestamps from the CompuScope digitizer allowed simple and accurate dead time corrections, $<5 \%$, to account for the lack of digitization during buffer readout. Neutrons were identified using pulse shape discrimination, and measurements were only performed in energy ranges where PSD was sufficient to avoid any significant overlap of the gamma and neutron distributions. Background measurements were taken for each sample; background neutron rates were a few percent of the rates with the source present. Although the focus of this study was on the relative change of efficiency with length and energy range, a series of measurements varying source standoff from 45 to $75 \mathrm{~cm}$ showed good $1 / \mathrm{r}^{2}$ dependence indicating that room return was not a large contribution, so absolute values of intrinsic efficiency are reported.

Scintillator light decays were measured with the Time Correlated Single Photon Counting (TCSPC) technique, using two PMTs. One PMT (Hamamatsu H2431-50) was closely coupled to a sample to capture as much of the scintillation light as possible, the other PMT (Hamamatsu 
H5784) was loosely coupled to observe not more than a single photon per interaction. Both PMTs were connected to constant fraction discriminators, and the time difference for each event, as determined by a Caen V1290N Time to Digital Converter, was added to a histogram. The decay spectrum was then fitted by sum of exponentials convolved with a Gaussian function for the instrument response. From the extracted decay constants and amplitudes, a decay spectrum was constructed showing the remaining fraction of the scintillation light for each point in time.

\section{Results and discussion}

A faceted solution-grown trans-stilbene crystal with a size of about $11 \mathrm{~cm}$ along the monoclinic b-axis is shown in Figure 1 A. Clear images of the "LLNL" text placed under the crystal show high optical transparency. The crystal does not have any visible defects except for slight traces of a long seed used to initiate crystal growth [17]. The lack of the residual stress that could be caused by the crystal growth procedure was demonstrated in that the crystal could be easily handled to obtain optical and scintillation parts of different shapes. A cylinder shown in Figure $1 \mathrm{~B}$ was produced by machining (lathing). Similar cylindrical samples used for characterization in this work were prepared manually using diamond wire saw cutting and standard methods of polishing.

$\mathrm{X}$-ray diffraction (XRD) analysis showed identical structures for solution- and meltgrown crystals (Figure 2), confirming the absence of solvent incorporation into the crystallographic lattice. The density of solution-grown crystals was measured as $1.16 \mathrm{~g} / \mathrm{cm}^{3}$, which is equal to that listed in the Birks's monograph [6]. These results, in combination with the close value for the melting point $\left(\sim 124{ }^{\circ} \mathrm{C}\right)$, indicate that solution-grown and traditional melt- 
produced stilbene represent the same material, which should have identical optical and scintillation properties unless they are altered by different impurities or structural imperfections.

\subsection{Scintillation light efficiency}

As reported previously [17], when pure stilbene material is used for crystal growth, the photoluminescence (PL) spectra of solution-grown crystals coincide with those of melt-grown stilbene. Figure 3 present the PL spectra of a 1-cm thick stilbene crystal measured in two different positions relatively to the excitation beam. The reflection spectrum corresponds to the excitation occurring only in a thin surface layer, so that the emitted light is little affected by the self-absorption of the crystal. This is a general spectrum close to that of the compound molecular spectrum. For scintillation applications, in which excitation and emission occur in the volume of the material, the transmission spectrum measured through the sample thickness has more practical importance, because it takes into account absorption of the emitted light by the molecules arranged into the crystallographic lattice. The resulting spectrum of the light collected by the PMT misses its shorter wavelength fraction, so that the peak energy of the transmission spectrum has a longer wavelength that, due to the increasing self-absorption, gradually red-shifts with the increasing crystal size.

Evaluation of the scintillation light efficiency of the solution-grown crystals was made by measurements relative to two ScintiTech-originated anthracene crystals provided by Sandia National Lab. In contrast to stilbene, which is stable in air for an indefinite duration of time, anthracene is known to be sensitive to easy surface oxidation. To avoid the effect of the degraded surface, both anthracene crystals, which had dimensions of $\sim 4 \mathrm{~cm}$ in diameter and $1 \mathrm{~cm}$ 
thickness, were polished prior to the measurements. Stilbene crystals used for comparison were cut to precisely the same dimensions and shapes to avoid any size-effect in the measurements made with the same ${ }^{137} \mathrm{Cs}$ source, PMT, and geometry of the experimental set-up. Results obtained by integration within the time gates selected to take into account the difference in the scintillation decays, showed the ratio of stilbene/anthracene LO detected by the PMT as 0.94 and 0.88, for two pairs containing anthracene crystals of different structural perfection. Corrections by a factor of 1.37 determined by comparison of the spectral ranges for anthracene luminescence and spectral sensitivity of the PMT brought these ratios to 0.69 and 0.64 , respectively. The relative light yield, estimated in these measurements is similar to the most reliable data reported for melt-grown crystals of different sizes $(\sim 0.6-0.7[24,25])$ and close to the theoretical estimations, from which this ratio is not expected to be higher than 0.75 [6].

The results obtained from the relative LO measurements confirm that the method of production does not affect fundamental properties, and solution growth can be successfully applied for production of stilbene with high scintillation efficiency. However, data obtained from such measurements made with relatively small crystals can hardly be used for estimation of the efficiencies in large-scale scintillators, in which complex effects of self-absorption may lead to different degree of light loss. In the current work, the degree of the LO degradation occurring in stilbene crystals due to these effects was evaluated from measurements made with samples of different thickness. To eliminate additional effects introduced by sample geometry, all crystals were cut as cylinders of the same diameter but different length increasing from a few mm to $\sim 10$ $\mathrm{cm}$. Several measurements were made with each sample to evaluate the effects of wrapping and mounting on the PMT, which produced variations within $\sim 3 \%$. Maximum values measured for individual samples were used for the final comparison. The results are illustrated by Figure 4, 
which presents the experimental dependencies of the measured LO on the length of the crystal cylinders. As shown by the results of Figure 4 A, while all measured stilbene crystals appeared brighter than common liquid scintillators, solution-grown samples exhibited noticeably higher LO in comparison to both liquids and melt-grown crystals. The difference was especially pronounced at larger size, where the solution-grown crystals might have close to twice the LO as same size melt-grown and liquid samples. A general tendency for all scintillators was the gradual decrease of the light intensity with the increasing size. However, the slopes of the dependences for two types of crystals were different, indicating smaller light loss with increasing size for the solution-grown samples. This observation is additionally illustrated by Figure $4 \mathrm{~B}$ that presents the LO normalized to the values obtained by the extrapolation of the linear dependences of Figure 4 A to the "zero" length. Simple estimations based on these dependences show that at 10 $\mathrm{cm}$ length solution-grown stilbene loses only $20 \%$ of its initial light, compared to almost $\sim 50 \%$ in the same size melt-grown crystal. Assuming that the same linear dependence stays valid for larger scale crystals, the same $\sim 50 \%$ loss in solution-grown crystals would be expected at about twice longer size $(\sim 20 \mathrm{~cm})$.

The measured difference in the light attenuation could be attributed to different traceimpurities difficult to analyze in high-purity material used for production of both types of crystals. There are, however, observations that indicate that the light loss could also be influenced by specific structural defects. It is known that melt-grown stilbene crystals are characterized by a mosaic structure, with varying degrees of block disorder [24], common for low-symmetry monoclinic crystals grown by the Bridgman techniques. Such blocks are not typical for solution growth that proceeds mainly by dislocation mechanisms forming more homogeneous single crystal structures. None of the crystals are structurally perfect, but 
inhomogeneity caused in solution-grown crystals by faceted structure (growth sectors of different facets), or vicinal sectors formed by dislocations growth, produce distortions of much smaller degree than misaligned structural blocks in melt-grown crystals (Figure 5). The mosaic structure of the melt-grown stilbene can be clearly seen by the naked eye because of the light scatter from the mis-oriented blocks and grain boundaries. When small samples are compared, there is almost no difference in the optical transparency of the crystals grown by the two different methods. However, as is illustrated by the photos of Figure 6, at larger size the light propagation becomes more distorted by the structural imperfections present in the melt-grown crystals. The effect can be easily understood taking into account the symmetry of stilbene, which determines its optical anisotropy and birefringence. Considerable scatter by the grain boundaries leads to longer paths of the scintillation light that loses its fraction to the additional selfabsorption. Due to the absence of similar grain boundaries, the effects of scatter and light loss are less pronounced in solution-grown crystals, explaining higher LO and the widening LO gap between the two crystal types at increasing size.

\subsection{Pulse shape discrimination}

Figure 7 shows examples of the original experimental data and neutron/gamma distributions used for PSD studies of crystals. In the higher electron-equivalent energy ranges, the results indicate more efficient PSD. At lower energy PSD degrades, primarily due to the decreasing number of photoelectrons that leads to larger fluctuation, broadening of the spectra, and overlap of the gamma and neutron distributions [26]. 
Analyses made for many individual crystals in different electron-equivalent energy ranges from 30 to $1000 \mathrm{keV}_{\text {ee }}$ showed a typical rise of PSD FoM $v s$ energy illustrated by Figure 8 for the case of the $75 \mathrm{~mm}$ long samples. The data also indicated a noticeable difference in PSD produced by crystals of different origin. Similar results were obtained in all other experiments that showed that, at equal dimensions, solution-grown crystals exhibit higher degree of discrimination in all energy ranges. To consider the effect of the increasing size, PSD FoMs for crystals of different lengths were calculated and compared in the same energy ranges. Figure 9 shows the results obtained with two types of crystals and EJ-309 liquids used as a common PSD reference. As follows from the data, all measured samples exhibit some degree of PSD decrease typical for the scintillators of the increasing size. However, while performance of solution-grown crystals remains substantially higher compared to the liquid scintillators at all measured dimensions, the advantage of melt-grown stilbene over the liquids seems to degrade at increasing scale. The effect is especially pronounced in the range of low energies (Figure $9 \mathrm{~B}$ ) where the melt-grown crystals show faster deterioration of discrimination at the increasing size. It could be argued that data collected in the present paper might be not sufficient to make final conclusion about the relative performance of crystals grown by two different techniques. While all tested solution samples were obtained under the same conditions from material of equal purity, crystals grown from melt were purchased at different times, and there can be considerably more fluctuations in their origin and quality. Additional uncertainties could be introduced by mixed measurements of bare and encapsulated crystals. The results of the current research convincingly show that PSD in solution-grown crystals is at least not worse than in previously known traditional stilbene. However, without any intention to diminish the importance of prior works conducted with the melt-grown crystals, some speculations can be made to suggest why, similar 
to the LO, crystals grown from solution should have better discrimination properties. The presence of impurities can be a strong contributing factor, affecting both the prompt and delayed components of scintillation light $[6,27]$. Much less information is available about the effects of structural imperfections that may also influence discrimination properties. It has been known [6] that, similarly to the effect of impurities, structural defects may reduce the exciton life time, leading to alteration of the decay characteristics that otherwise would be constant for a given material. Since both impurities and lattice defects work as absorption or exciton trapping sites, their presence in crystals should lead to the reduction of the excitation life times. This may explain discrepancies in the values measured for the same materials of different origins. Such assumption is in agreement with the results of our TCSPC measurements that produced decay characteristics corresponding to the following multi-exponential fits for both types of crystals:

$$
\begin{gathered}
f(t)_{\text {Solution }}=0.872 e^{-\frac{t}{4.3 n s}}+0.074 e^{-\frac{t}{34.8 n s}}+0.054 e^{-\frac{t}{332 n s}} \text { and } \\
f(t)_{\text {Melt }}=0.868 e^{-\frac{t}{3.6 n s}}+0.076 e^{-\frac{t}{31 n s}}+0.056 e^{-\frac{t}{310 n s}}
\end{gathered}
$$

The results indicate a typical $3.6 \mathrm{~ns}$ [25] prompt decay for the melt-grown stilbene and longer 4.3 ns for the crystals grown from solution. The presence of grain boundaries acting as traps could also lead to a partial loss of excitation that should be more pronounced for the long-living triplet states than for the shorter lived excited singlets. In organic scintillators, the initial singlet excitations produce the majority of the light output, while the number and kinematics of the excited triplet states control the amount of the delayed light in the scintillation pulses. The increasing proportion of the triplet excitations lost on the structural defects may lead to a larger decrease of the delayed light fraction, explaining more pronounced deterioration of PSD in large melt-grown crystals. 


\subsection{Neutron detection efficiency}

As shown by the experimental results of PSD measurements (Figure 7 A), a large fraction of incident neutrons from a fission spectrum produce counts in the low electron-equivalent energy ranges close to the limit of the complete overlap of neutron and gamma distributions. Statistical analysis shows that, when measurements are made with unshielded ${ }^{252} \mathrm{Cf}$ source, the range of 48 $170 \mathrm{keV}_{\mathrm{ee}}$ has approximately as many neutron counts as the much wider range from $170 \mathrm{keV}_{\mathrm{ee}}$ to $2000 \mathrm{keV}_{\text {ee. }}$ This makes enhancement of PSD in the range of lower energies one of the important factors benefiting the overall neutron detection efficiency. The limit corresponding to the low-energy PSD cuts depends on many factors that include the scintillation LO, crystal shape and dimensions, experimental set-up geometry, etc. One of the ways for defining these limits for different crystals could be determination of the energies corresponding to the minimal separation between normal neutron and gamma distributions, S. However, for practical reasons, it would seem more logical to conduct the comparison at some equal low mean PSD that still can be suitable for efficient discrimination of the counts. Similar to a previous studies [14], such a low limit for efficient discrimination was selected at the separation between two Gaussian distributions corresponding to $S>3\left(\sigma_{\text {gamma }}+\sigma_{\text {neutron }}\right)$, where $\sigma$ is the standard deviation for each peak. Based on this selection, all crystals tested in the present work were compared at equal FoM $=1.27$ corresponding to $3 \sigma$ separation defined as the low energy cuts for efficient PSD. Figure 10 presents a comparison of the low energy cuts obtained for the tested crystals of different length. Each point shown on the graph correspond to the PSD FoM=1.27. However, 
the electron-equivalent energy at which samples produce equal discrimination of $3 \sigma$ is different for crystals of different length and origin. Comparison of the data suggests that the use of solution-grown crystals may enable the achievement of lower energy limits at the same effective discrimination. The advantage again becomes especially considerable at increasing scintillator length. Assuming that quenching factors reported for stilbene in previous papers $[20,21]$ are applicable to the $10 \mathrm{~cm}$ long crystals tested in the present studies, effective $3 \sigma$ separation between neutron and gamma peaks can be obtained with the melt grown crystals at corresponding recoil proton energies above $\sim 650 \mathrm{keV}$, while the use of the same size solutiongrown crystals may decrease this limit to about $450 \mathrm{keV}$. Figure 11 illustrates the effect of these differences on the overall neutron detection efficiency calculated for crystals of different length. The efficiency should be the same for melt and solution grown crystals, regardless of optical or PSD performance, over the same gamma equivalent energy range, if the identical chemical composition, density and quench response are retained by both growth methods. This is occuring

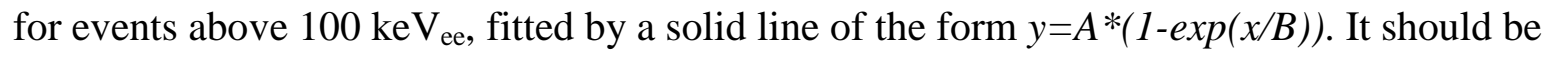
noted here, that despite this equality of efficiencies measured at high energy ranges, the higher degree of PSD obtained with the solution-grown crystals (Figure 9) should lead to the increased gamma rejection, an extremely important parameter of any detector performing on the basis of pulse shape discrimination. If the discrimination threshold is selected for each crystal at energy coincident with FoM=1.27, the corresponding gain in detection of recoil protons with lower energy leads to the increased neutron detection efficiency for solution-grown crystals (dashed lines of Figure 11). There will be a similar benefit regardless of the FoM chosen to define the low energy bound. 
The data on neutron efficiencies collected in these studies with relatively small size and number of crystals in an environment not fully characterized for room return should be considered as preliminary. If the research continues with crystals available at larger size and quantities, more investigations are needed to verify quenching factors that may be affected by size and geometry, as well as to define the discrimination parameters which might produce better performance in the range of lower energy. Additional studies should be made for more accurate comparison of crystals to large scale liquid scintillators to define benefits of stilbene for potential applications in high efficiency neutron detection systems.

\section{Conclusion}

Scintillation properties of trans-stilbene single crystals grown to a scale exceeding $10 \mathrm{~cm}$ by application of the solution growth technique were investigated and compared to the performance of similar crystals obtained by traditional methods of melt-growth. Results showed that under the condition of high purity raw materials, solution growth produces single crystals of the same crystallographic symmetry, with basic physical properties known for trans-stilbene crystals for many decades. Comparative studies conducted with crystals of different length showed that the new material has light output and pulse shape discrimination better than traditional melt-grown stilbene or liquid scintillators of the same size. Measurements made with $51 \mathrm{~mm}$ diameter scintillators showed that at the length $\sim 10 \mathrm{~cm}$ the light output of solution-grown stilbene can be almost twice higher than that of the liquid scintillator EJ-309 and about 50\% higher than the light output of the tradition melt-grown stilbene. Similar correlations were 
obtained in PSD studies that resulted in $30-40 \%$ higher PSD FoM values for solution-grown crystals in comparison to both liquid scintillators and melt-grown stilbene. Preliminary analysis also showed that the use of solution-grown crystals has potential for achieving higher degree of discrimination in low energy ranges, important for the enhancement of neutron detection efficiency.

The technology for solution growth of $10 \mathrm{~cm}$ scale stilbene crystals has been transferred to Inrad Optics [23] for commercial production in the US. The next step of the research is planned to be conducted with larger-scale crystals that may become available by further advancement of the solution growth technique and application of rapid growth methods developed previously for inorganic crystals grown from aqueous solutions [28]. The goal of this development will be reproducible production of larger volumes at a substantial decreased cost to finally make stilbene single crystals available for wide-spread use in neutron detection.

\section{Acknowledgment}

The work was performed under the auspices of the US Department of Energy by Lawrence Livermore National Laboratory under ContractDE-AC5 07NA27344. Financial support was provided by the US Department of Homeland Security, Domestic Nuclear Detection Office (DNDO), under competitively awarded IAA HSHQDC-09-X-00743. This support does not constitute an express or implied endorsement on the part of the Government. We also wish to thank Dr. Cheng Saw for performing X-ray diffraction analysis and Dr. Erik Brubaker for providing the anthracene crystals. 


\section{References}

1. G. T. Wright, Proc. Phys. Soc. B 69 (1956) 358-372.

2. R. B. Owen, I.R.E. Transactions on Nuclear Science, NS-5, No.3 (1958) 198-201.

3. F. D. Brooks, Nucl. Instr. Meth. 4 (1959) 151-163.

4. R. C. Sangster and J. W. Irvine, J. Chem. Phys. 24 (1956) 670-715.

5. L. M. Bollinger and G. E. Thomas, Rev. Sci. Instrum. 32 (1961) 1044-1050.

6. J. B. Birks, The Theory and Practice of Scintillation Counting. London, U.K.: Pergamon, 1964.

7. D. L. Horrocks, Appl. Spectroscopy 24 (1970) 397-404.

8. Yu. A. Kaschuk, B. Esposito, L. A. Trykov, V. P. Semenov, Nucl. Instr. Meth. A 476 (2002) $511-515$

9. E. E. Klimenkov, Yu. A Kashcuk, A. V. Krasilnikov, A. A. Oleinikov, V. D. Sevastyanov, L. A. Trykov, and N. N. Semenova, Instruments and Experimental Techniques 47 (2004) 166167. Translated from Pribory i Tekhnika Eksperimenta, No. 2, 2004, 35-37.

10. N. Z. Galunov, E. V. Martynenko, O. A. Tarasenko, Functional Materials 13 (2006) 359364.

11. L. F. Nakae, G. F. Chapline, A. M. Glenn, P. L. Kerr, K. S. Kim, S. A. Ouedraogo, M. K. Prasad, S. A. Sheets, N. J. Snyderman, J. M. Verbeke, R. E. Wurtz, AIP Conference Proceedings, 1412 (2011) 240-248. 
12. Ch. A. Hagmann, D. D. Dietrich, J. M. Hall, P. L. Kerr, L. F. Nakae, R. J. Newby, M. S. Rowland, N. J. Snyderman, and W. Stoeffl, IEEE Trans. Nucl. Scie. 56 (2009) 1215-1217.

13. S. V. Budakovsky, N. Z. Galunov, N. L. Karavaeva, J. K. Kim, Y. K. Kim, O. A. Tarasenko, and E. V. Martynenko, IEEE Trans. Nucl. Sci. 54 (2007) 2734-2740.

14. N. Zaitseva, B. L. Rupert, I. Pawelczak, A. Glenn, H. P. Martinez, L. Carman, M. Faust, N. Cherepy, S. Payne, Nucl. Instrum. Meth. A 668 (2012) 88-93.

15. A. Hoekstra, P. Meertens, and A. Vos, Acta Cryst. B31, (1975) 2813-2817.

16. N. P. Zaitseva, J. J. DeYoreo, M. R. Dehaven, R. V. Vital, K. E. Montgomery, M. Richardson, and L. J. Atherton, J. Cryst. Growth 180 (1997) 255-262.

17. L. Carman, N. Zaitseva, H. P. Martinez, B. Rupert, I. Pawelczak, A. Glenn, H. Mulcahy, R. Leif, K. Lewis, S. Payne, J. Cryst. Growth 368 (2013) 56-61.

18. Scintillation Technologies, http://www.scintitech.com.

19. ProChem Inc, http://www.prochemonline.com.

20. R. L. Craun and D. L.Smith, Nucl. Inst. Meth. 80 (I970) 239--244

21. Y. Shimizu, M. Minowa, H. Sekiya, Y. Inoue, Nucl. Inst. Meth. A, 496 (2003) 347-352.

22. N. Zaitseva, A. Glenn, H. P. Martinez, L. Carman, I. Pawełczak, M. Faust, and S. Payne, Nucl. Inst. Meth. A, 729 (2013) 747-754.

23. Inrad Optics, http://www.inradoptics.com

24. N. Z. Galunov, O. A. Tarasenko, V. A. Tarasov, Functional Materials 20 (2013) 304-309. 
25. Amcrys-H, http://www.amcrys-h.com

26. D. L. Horrocks, Applied Spectroscopy 24 (1970) 397-404.

27. N. Zaitseva, L. Carman, A. Glenn, R. Hatarik, S. Hamel, M. Faust, B. Schabes, N. Cherepy, S. Payne, IEEE Trans. Nucl. Sci. 58 (2011) 3411-3420.

28. N Zaitseva, L. Carman, Rapid growth of KDP-type crystals, Progress in Crystal Growth and Characterization of Materials 43 (2001) 1-118. 


\section{Figure Captions}

Figure 1. Images of solution grown trans-stilbene: A - "as grown" faceted crystal with a label clearly seen through the $\sim 60 \mathrm{~mm}$ thickness; B - a 50-mm diameter cylinder manufactured by machine lathing from a faceted crystal (courtesy of Inrad Optics [23]).

Figure 2. XRD patterns of stilbene powders obtained from solution- and melt-grown stilbene crystals. Complete coincidence of reflection peak positions for both types of crystals indicates identical crystallographic structure coinciding with that listed for transstilbene by the International Center for Diffraction data (ICDD).

Figure 3. Photoluminescence spectra obtained with a $1 \mathrm{~cm}$-thick plate cut from a solution-grown stilbene crystal. In reflection mode, excitation occurs only in a thin surface layer. The transmission spectrum corresponds to the fraction of the emission light coming out of the crystal volume. Excitation wavelength in both cases was $273 \mathrm{~nm}$.

Figure $4 .{ }^{137} \mathrm{Cs}$ - un-normalized scintillation light output (LO) measured in solution- and meltgrown stilbene crystal cylinders of equal diameter $(51 \mathrm{~mm})$ : A - LO decrease with increasing crystal length; similar data measured with EJ-309 are shown to emphasize higher efficiency of both type of crystals in comparison to common liquid scintillators; 
B - stilbene LO normalized to the maximum value at "zero" length for each type of crystals.

Figure 5. Microscopic pictures showing more homogeneous single-crystal structure of solutiongrown stilbene in comparison with the structure of melt-grown crystals consisting of misaligned blocks separated by grain boundaries. The images of the plates cut perpendicular to the monoclinic b-axis were taken between crossed polarizers with a long focal distance microscope. The diameter of the circular field for both crystals is about $12 \mathrm{~mm}$. Arrow points to the tip of a vicinal sector typical for single crystals grown from solution by the dislocation mechanism.

Figure 6. Photographs of stilbene crystals taken with corresponding labels placed under the bottoms of 25- and 75-mm-long cylinders. Comparison shows that the difference in optical transparency, almost unnoticeable in small-scale crystals, becomes substantial at increasing size due to degrading light propagation caused by the larger amount of structural defects in the melt-grown crystals.

Figure 7. Examples of data used in PSD analyses of stilbene crystals exposed to ${ }^{252} \mathrm{Cf}$ : $\mathrm{A}-$ an experimental PSD pattern of a $75 \mathrm{~mm}$ long solution-grown stilbene obtained using the integration windows set to maximize the FoM for $450-500 \mathrm{keV}_{\text {ee }}$ energy range; $\mathrm{B}$ - 
corresponding PSD slice used for calculation of FoM in 450-500 $\mathrm{keV}_{\mathrm{ee}}$ energy range; C - similar PSD distribution obtained for a lower energy range of 40-50 $\mathrm{keV}_{\text {ee. }}$

Figure 8. Experimental dependences of PSD FoMs on electron-equivalent energy calculated for two $75 \mathrm{~mm}$ long stilbene crystals grown by different methods. Similar increase of PSD with the increasing energy was obtained with all stilbene cylinders irradiated by ${ }^{252} \mathrm{Cf}$ source. Individual FoMs were maximized by the variation of optimal start of the delayed integration window from $35 \mathrm{~ns}$ at $\sim 40 \mathrm{keV}_{\text {ee }}$ to $60 \mathrm{~ns}$ at $600 \mathrm{keV}_{\text {ee }}$ after the peak sample. The integration stops were kept constant (500 ns) for all calculations in order to simplify ADC conversion. The dashed lines in this and following pictures do not carry any physical meaning, being introduced only for convenience of representation.

Figure 9. Decrease of PSD FoM with increasing length obtained with $50 \mathrm{~mm}$-diameter stilbene cylinders in two different electron-equivalent energy ranges: $\mathrm{A}-480 \mathrm{keV}_{\mathrm{ee}} ; \mathrm{B}-75$ $\mathrm{keV}_{\mathrm{ee}}$. In addition to the larger FoMs calculated for all energy ranges, solution-grown crystals exhibit lower degree of PSD degradation with the scintillation length. Data measured for liquid scintillators are given for comparison to both types of crystals. The tail integration window used for calculation is $40 \mathrm{~ns}$ to $350 \mathrm{~ns}$ after the peak sample. 
Figure 10. Low energy limits corresponding to full $(3 \sigma)$ separation between neutron and gamma distributions calculated for stilbene scintillators of different length. The results calculated for each length within accuracy $\sim 5 \%$, show that solution-grown crystals enable detection and discrimination of lower energy pulses. The advantage becomes especially considerable at the increasing scintillator length required for higher neutron detection efficiency. The tail integration window used for calculation is 40 ns to 350 ns after the peak sample.

Figure 11. Neutron detection efficiency for an unshielded Cf-252 source obtained with stilbene scintillators of different length. The solid line is a fit to the data points corresponding to the pulses measured in the range of electron-equivalent energy above $100 \mathrm{keVee}$. The dashed lines connect respective experimental data obtained for the two types of crystals above the low energy cuts corresponding to FoM=1.27. The tail integration window used for calculation is $40 \mathrm{~ns}$ to $350 \mathrm{~ns}$ after the peak sample. 
Click here to download high resolution image
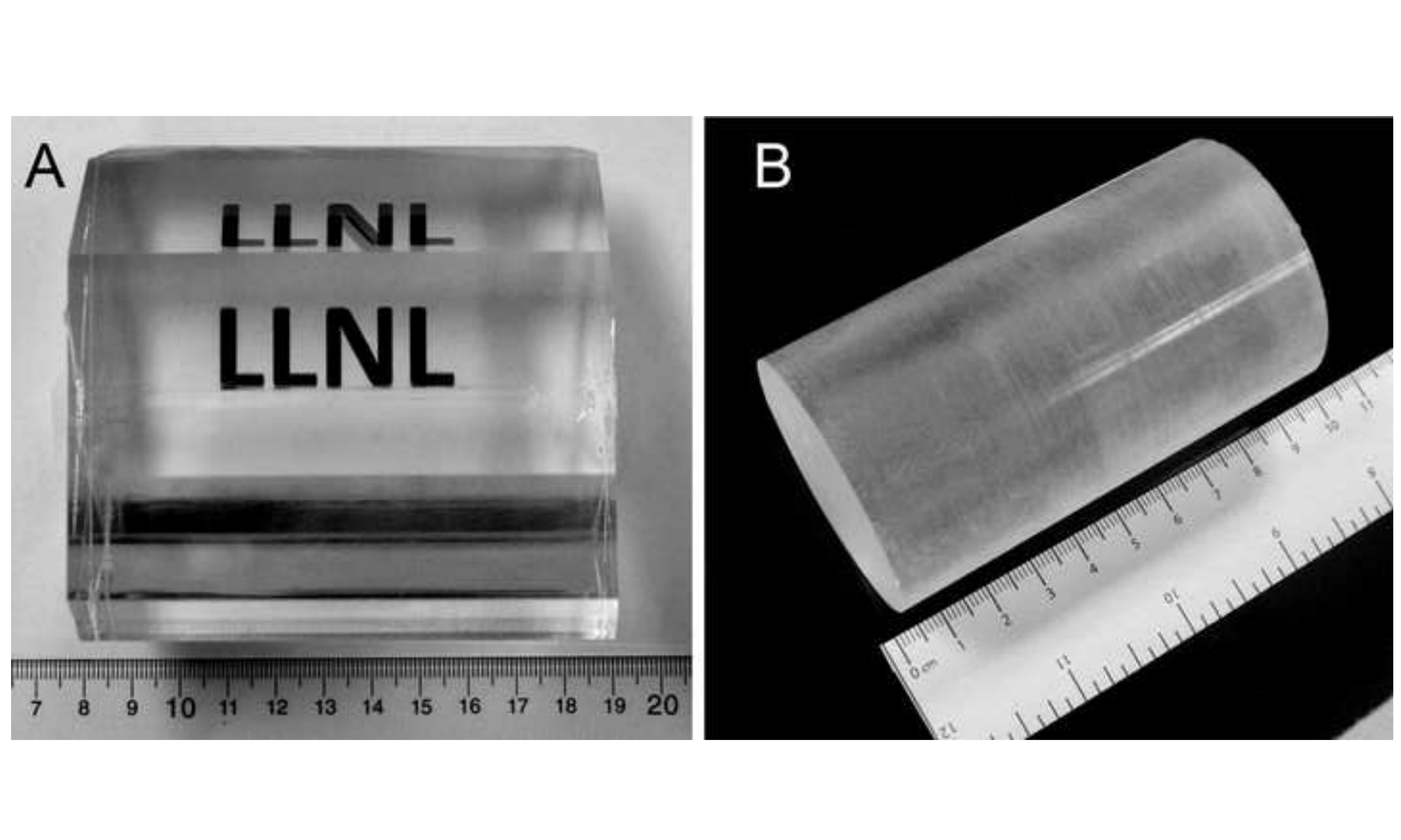


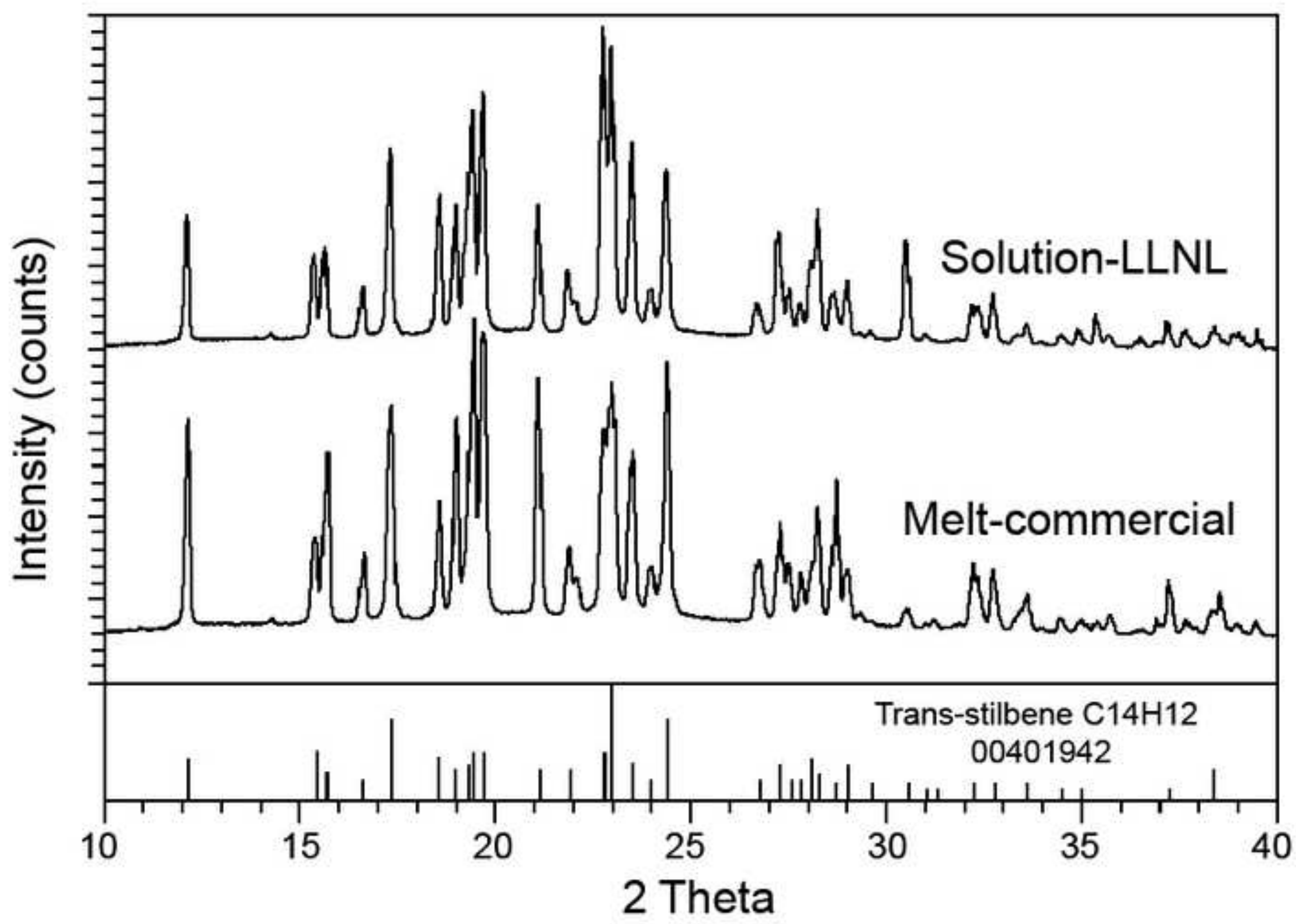




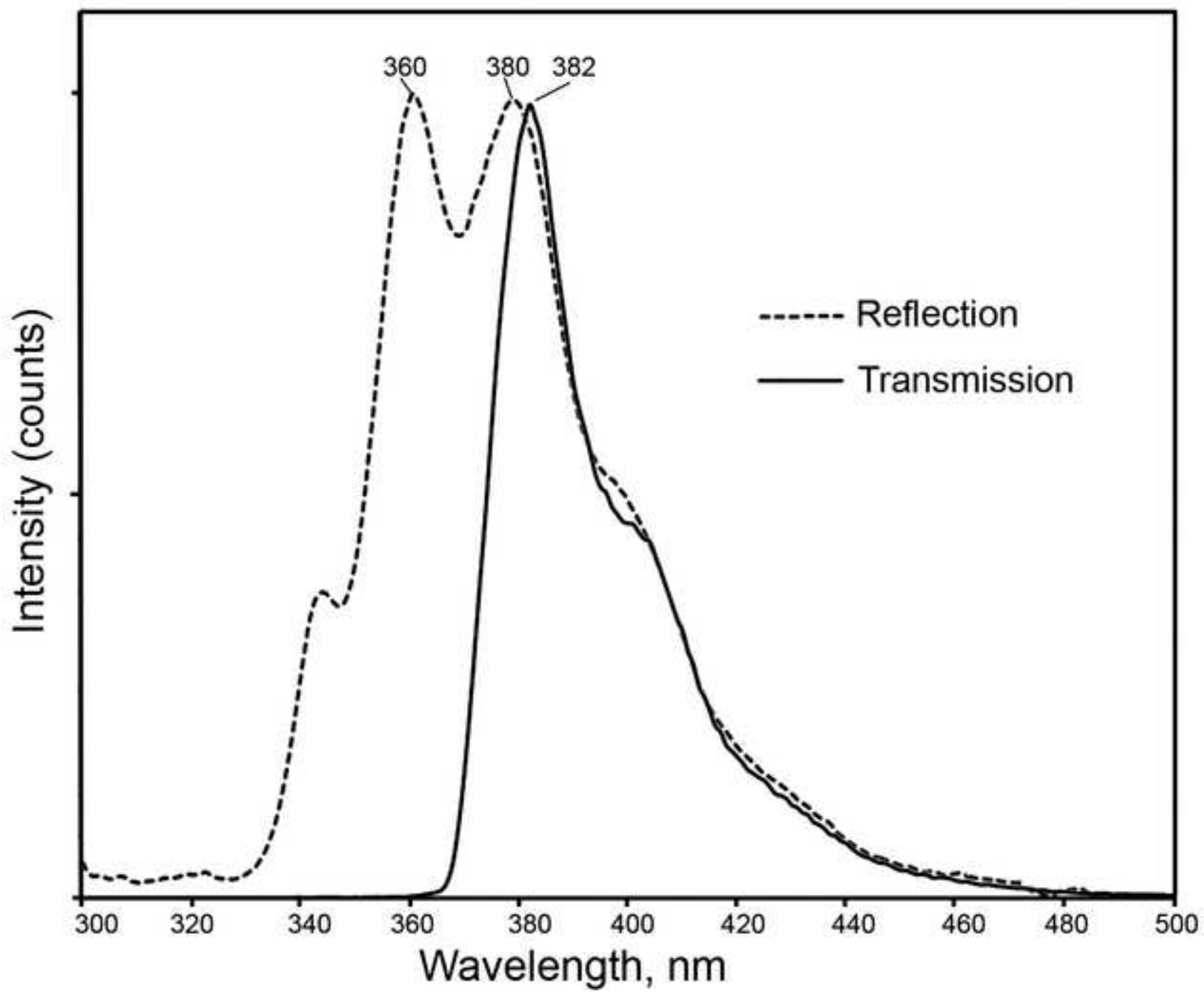



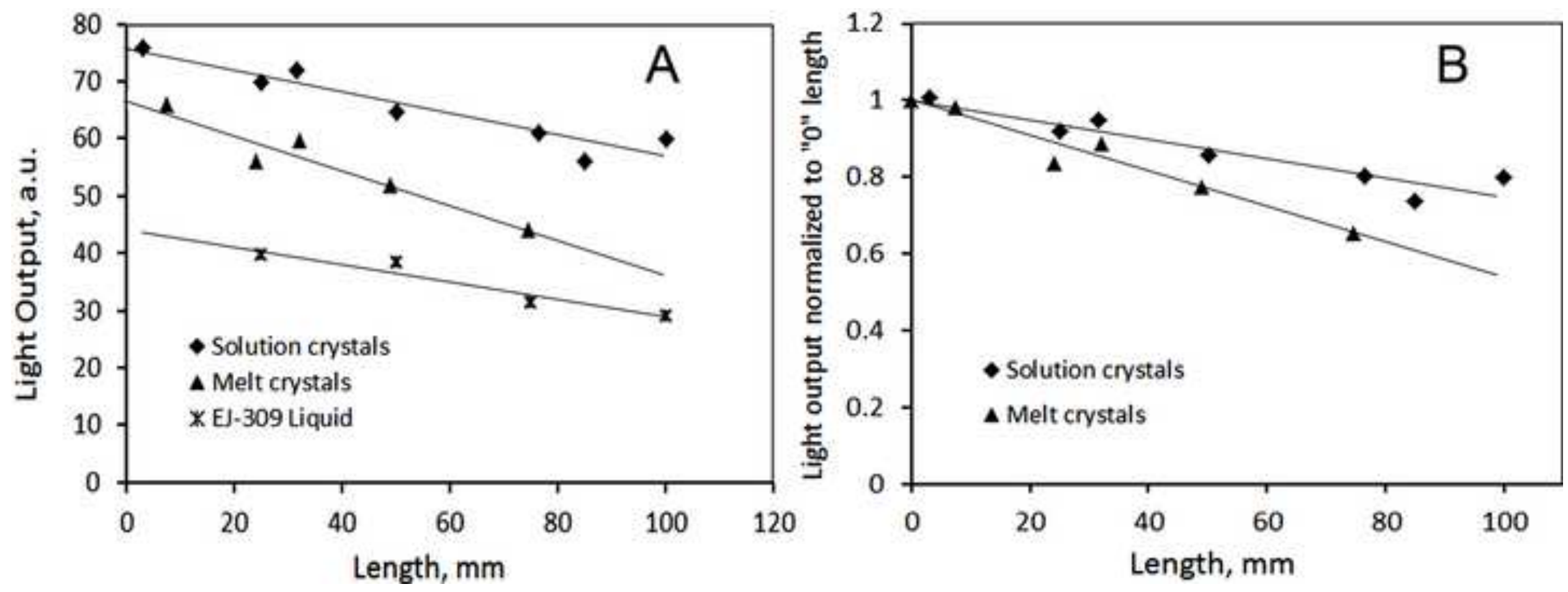


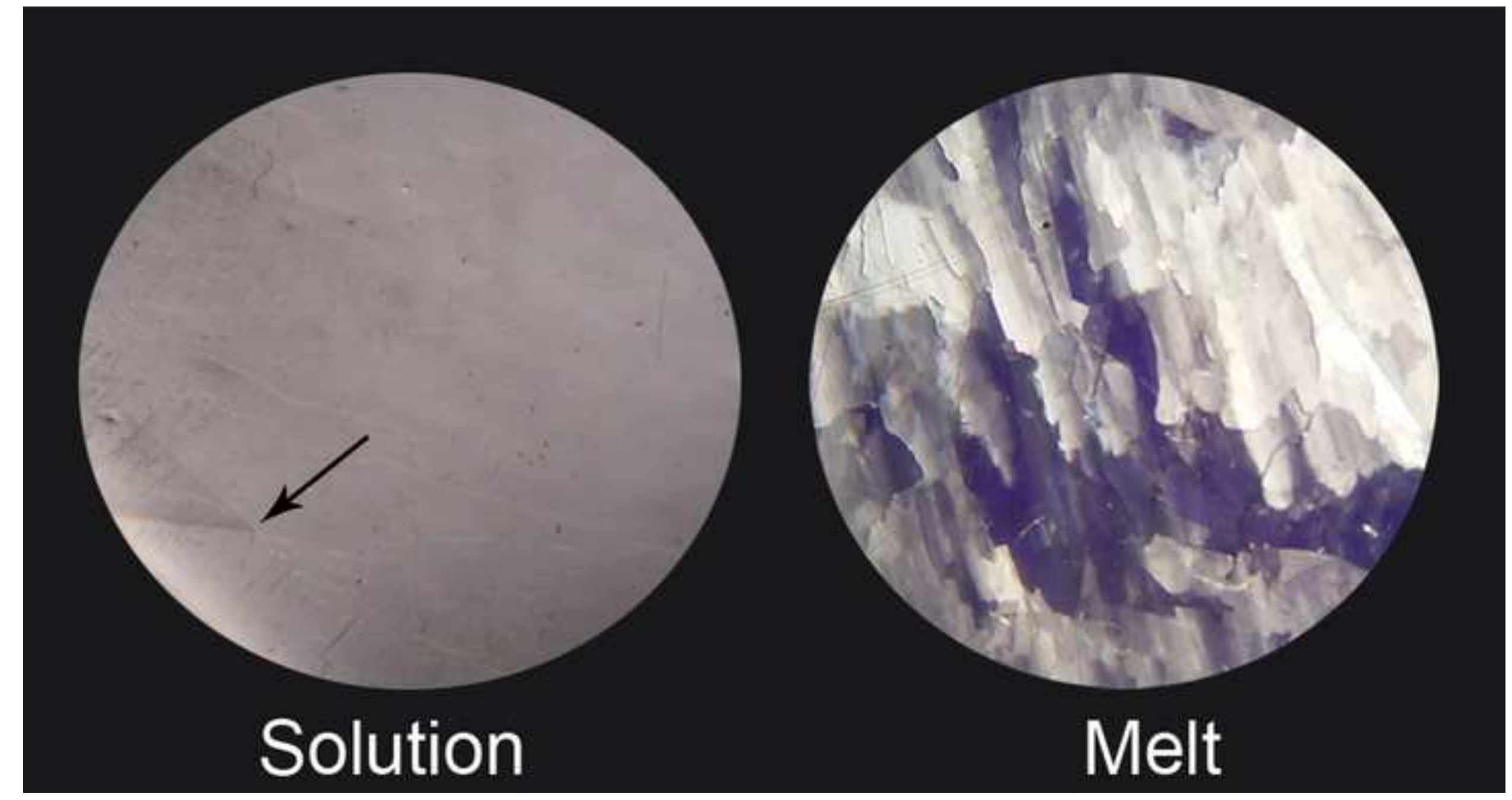

Solution 

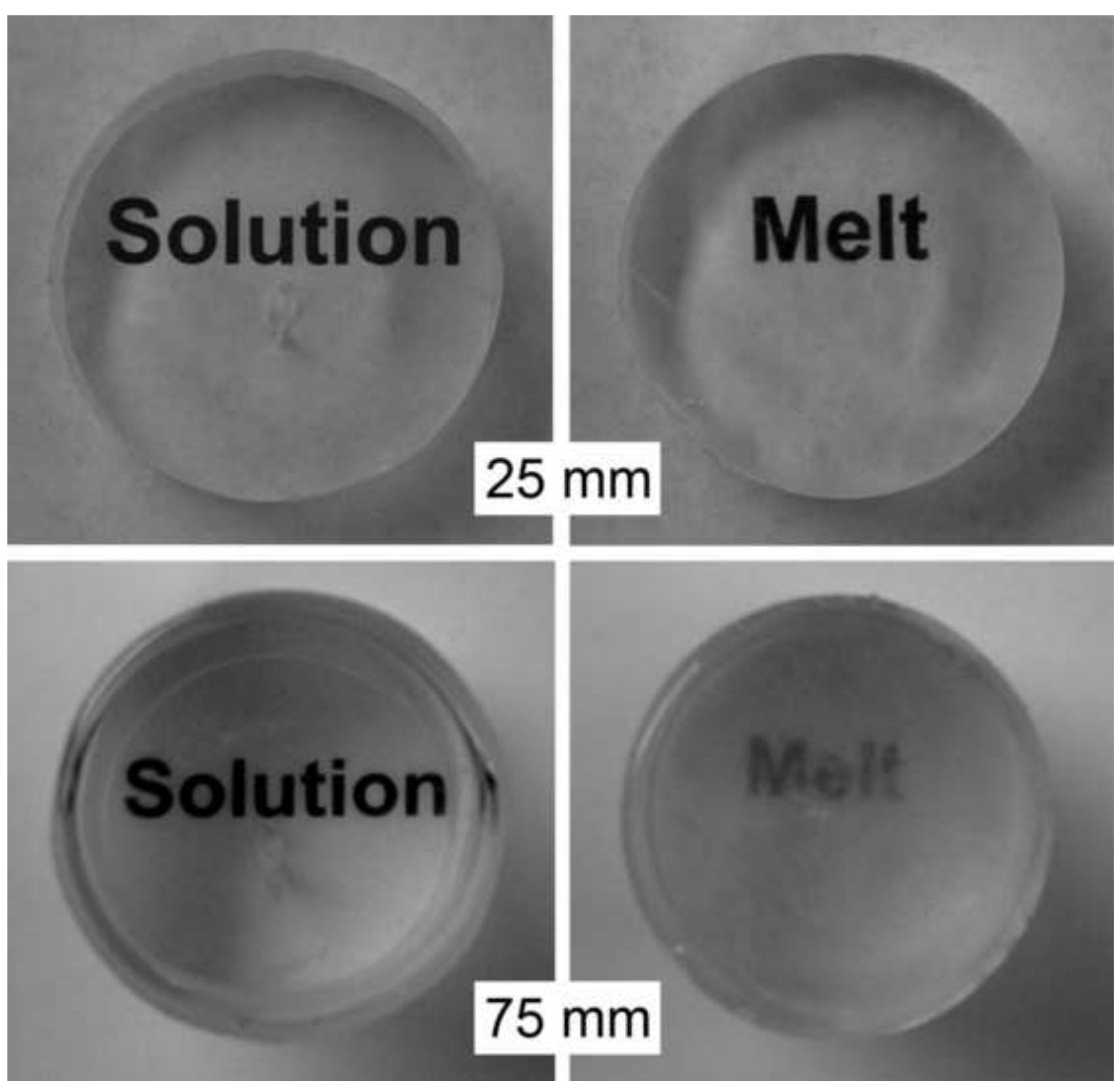

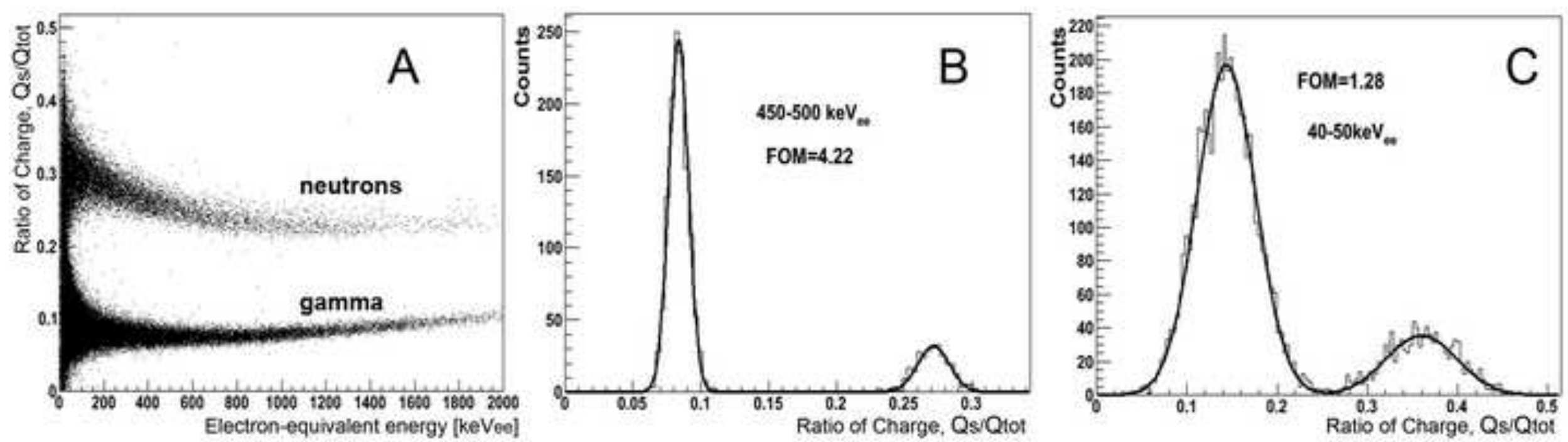


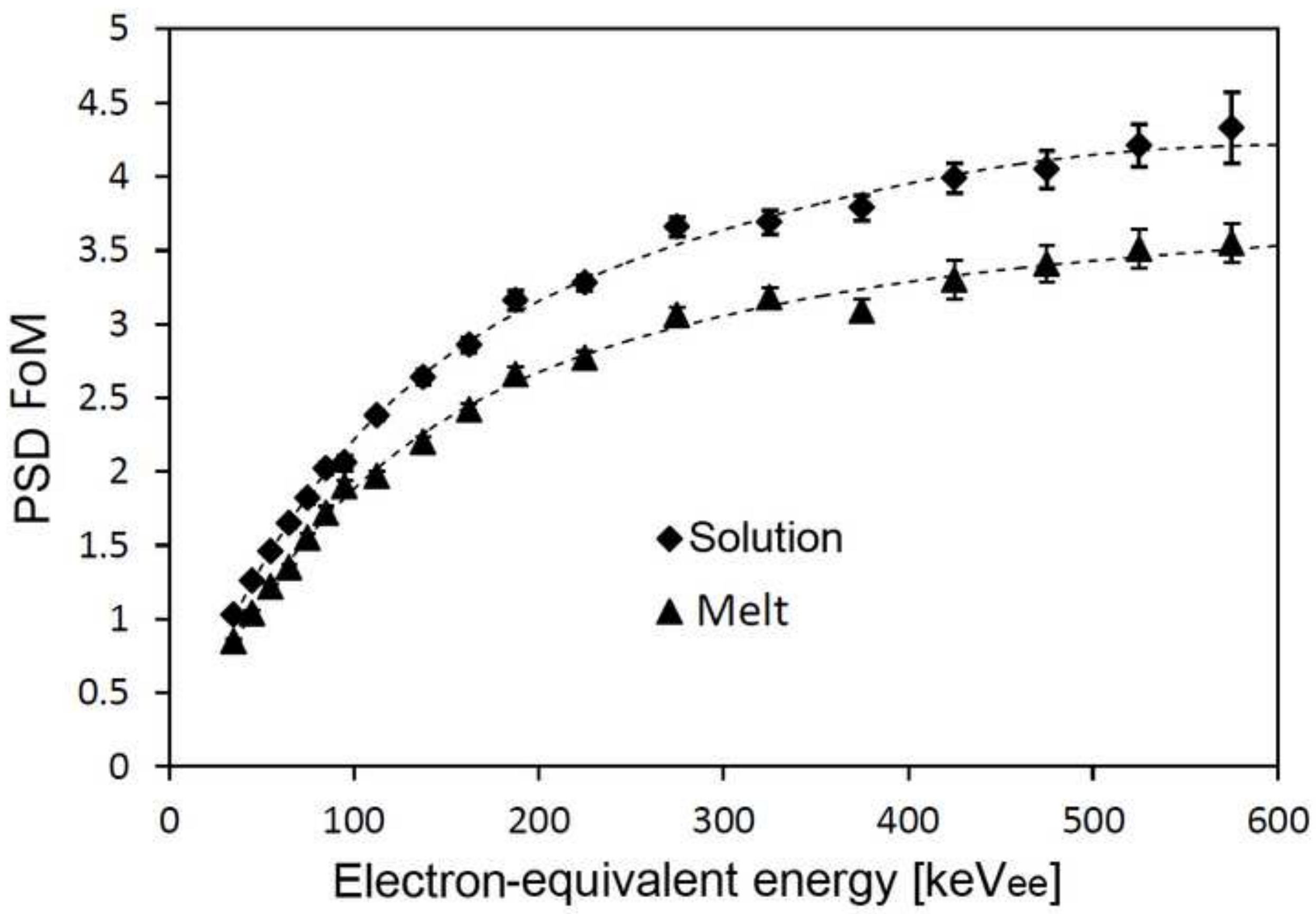



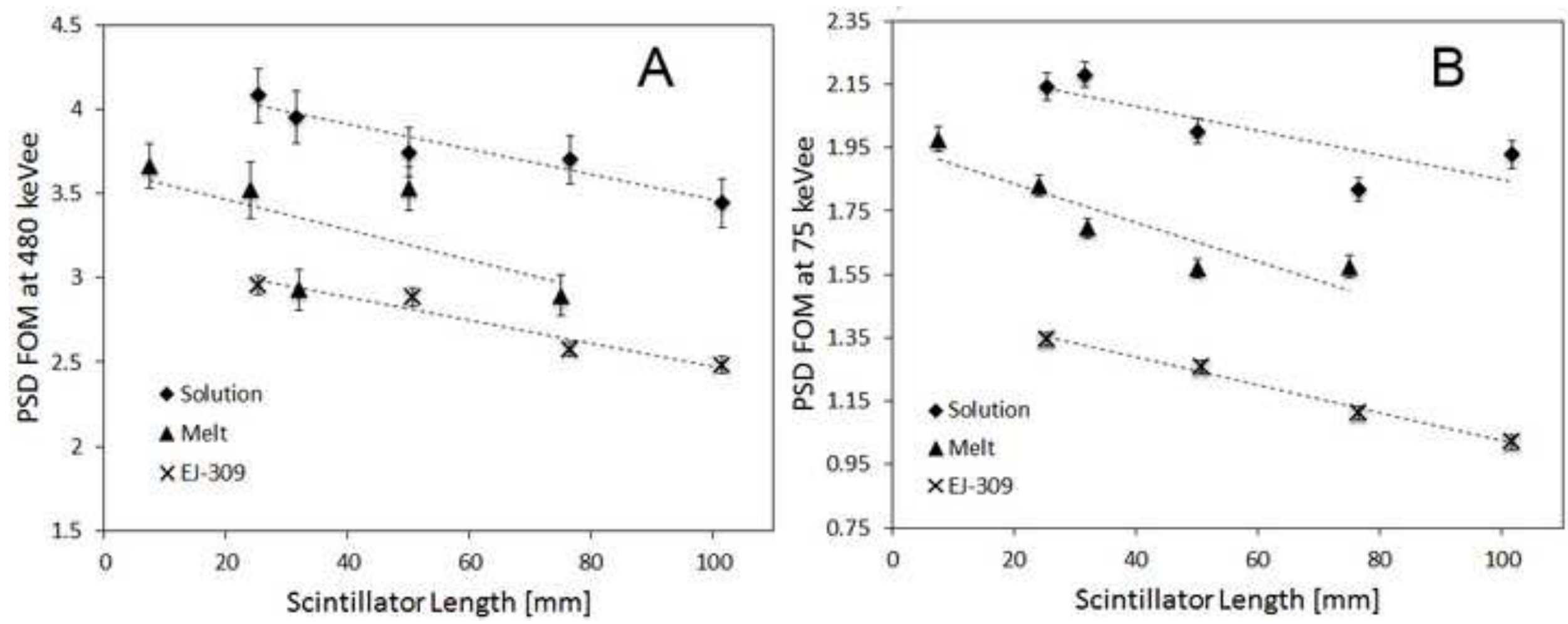


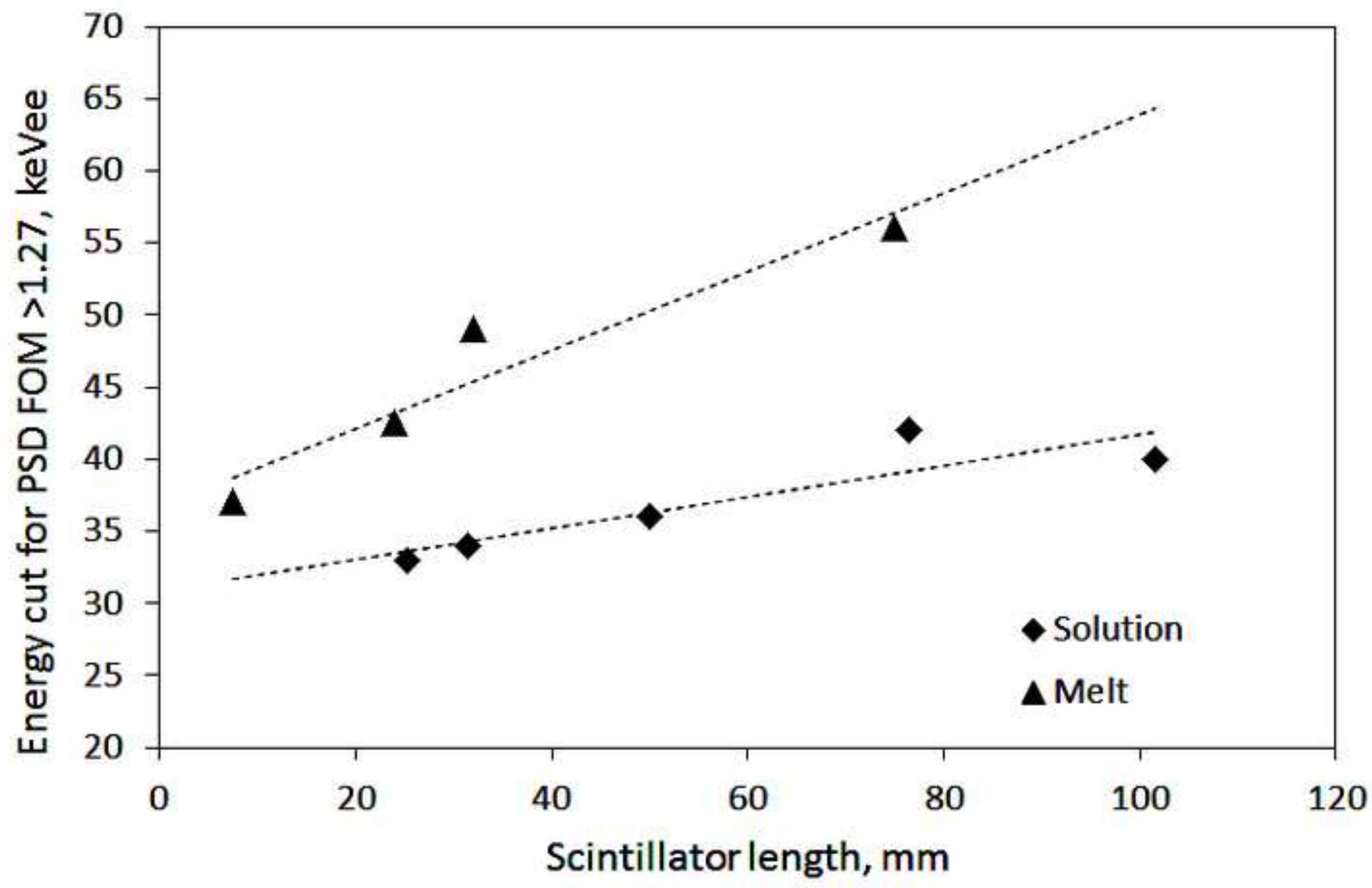




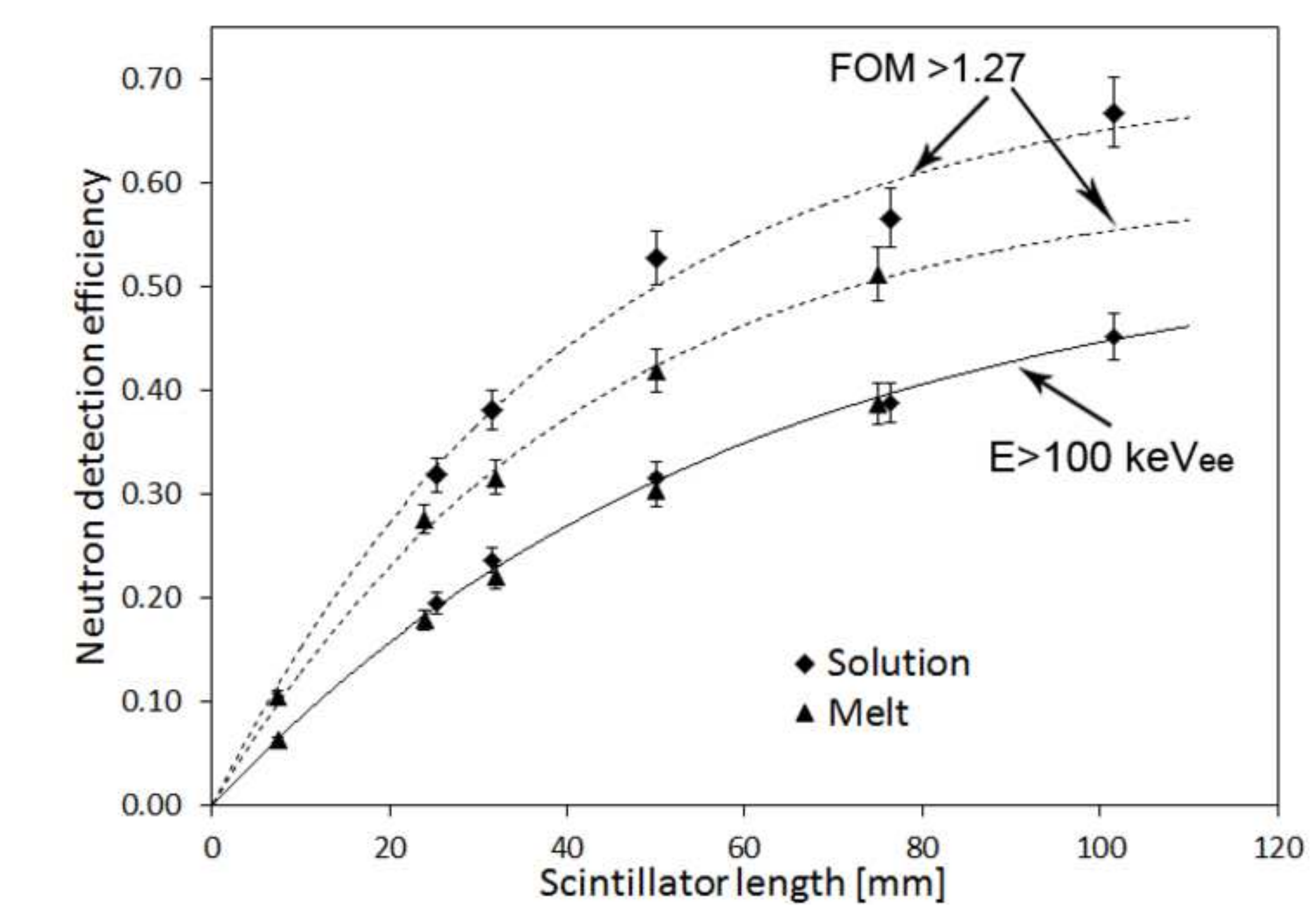

